\title{
Exploring biomarkers and therapeutic targets for pressure overload induced heart failure based on microarray data
}

\author{
Yongli He ${ }^{1 \#}$, Zhe Feng ${ }^{2 \#}$, Jianjun Lu $^{3,4}$, Rui Wang ${ }^{1}$, Cheng Huang ${ }^{1}$, Yingling Zhou ${ }^{1}$ \\ ${ }^{1}$ Guangdong Cardiovascular Institute, Guangdong Provincial People's Hospital, Guangdong Academy of Medical Sciences, Guangzhou, China; \\ ${ }^{2}$ The First Affiliated Hospital of Henan University of Science and Technology, College of Clinical Medicine of Henan University of Science and \\ Technology, Luoyang, China; ${ }^{3}$ The Second Clinical Medical School, Southern Medical University, Guangzhou, China; ${ }^{4}$ Department of Medical \\ Services, The First Affiliated Hospital of Sun Yat-sen University, Guangzhou, China \\ Contributions: (I) Conception and design: Y Zhou, C Huang; (II) Administrative support: Y Zhou, C Huang; (III) Provision of study materials or \\ patients: Y He, Z Feng; (IV) Collection and assembly of data: Y He, Z Feng, J Lu, R Wang; (V) Data analysis and interpretation: Y He, Z Feng, J Lu, \\ R Wang; (VI) Manuscript writing: All authors; (VII) Final approval of manuscript: All authors. \\ \#These authors contributed equally as the co-first author. \\ Correspondence to: Yingling Zhou; Cheng Huang. Guangdong Cardiovascular Institute, Guangdong Provincial People's Hospital, Guangdong \\ Academy of Medical Sciences, 96 Dongchuan Road, Guangzhou, China. Email: zylgdh@163.com; linfeng7822@sina.com.
}

Background: Heart failure (HF) is an end stage heart condition with poor prognosis which brings about tremendous social medical cost. Along decades, mechanism and treatments of HF have been under restless research.

Methods: In the present study, we first established pressure overload induced HF model using transaortic arch constriction (TAC) method in mice. The global expression profiles of long noncoding RNA (lncRNA), microRNA (miRNA) and messenger RNA (mRNA) were obtained by microarray probes, which were further confirmed by quantitative PCR (qPCR). Bioinformatics analysis was performed using multiple methods including volcano plotting, heatmapping and hierarchical clustering, Gene Ontology (GO) and pathway enrichment analysis, and competing endogenous RNA (ceRNA) regulatory network construction.

Results: Totally, 1,139 differentially expressed mRNAs (DEmRNAs), 3,830 lncRNAs (DElncRNAs) and 13 miRNAs (DEmiRNAs) were identified in HF group compared to control group, which could distinctly differentiate HF from normal control and were potential candidate biomarkers for HF. GO and pathway enrichment analysis revealed that multiple significant biological processes and pathways were involved in $\mathrm{HF}$ pathogenesis, such as extracellular matrix structural constituent, proteinaceous extracellular matrix, positive regulation of apoptotic process and integrin signaling pathway. Nine DElncRNAs, 3 DEmiRNAs and 25 DEmRNAs were filtrated out to construct a ceRNA network, which visually displayed their regulatory roles with therapeutic target potential.

Conclusions: The present study identified differentially expressed RNAs that might be involved in the pathogenesis and progression of HF. The outcomes shed lights into the underlying mechanisms for HF and provided candidate biomarkers and intervention targets for further research.

Keywords: Transaortic arch constriction (TAC); heart failure (HF); microarray; bioinformatics analysis; ceRNA regulatory network

Submitted Apr 28, 2020. Accepted for publication Aug 30, 2020.

doi: $10.21037 / \mathrm{cdt}-20-465$

View this article at: http://dx.doi.org/10.21037/cdt-20-465 


\section{Introduction}

Heart failure (HF) is a series of clinical symptoms resulting from systolic or diastolic cardiac dysfunction of various causes, as the heart cannot pump enough blood to meet the body's metabolic needs, which indicates poor prognosis (1). However, current tools fail to identify the early changes at cellular level in a failing heart, leaving early diagnosis and early treatment difficult to achieve. Thus, the exploration of novel biomarkers, regulatory mechanisms and targets of $\mathrm{HF}$ is much of urgency.

Noncoding RNAs (ncRNAs) are a group of RNA transcripts unable to code proteins but capable of finetuning genomic interactions to maintain biological hemostasis, which mainly include long noncoding RNAs (lncRNAs), microRNAs (miRNAs) and circular RNAs (circRNAs). Competing endogenous RNA theory is widely accepted as one of the most popular regulatory mechanisms among ncRNAs, which suggests lncRNAs or circRNAs have the effect of sponging miRNA via certain seed sequences called miRNA response elements (MRE), thus weakening the suppressive impact of miRNAs on mRNAs and affecting gene translation $(2,3)$. Growing number of studies have discovered specific lncRNAs acting as miRNA sponges regulating pathophysiological processes in cardiovascular diseases. Wang $\mathrm{K}$ reported that lncRNA CHRF sponged miRNA-489 to prevent hypertrophic responses both in vitro and in vivo after angiotensin II exposure (4). Similarly, it was uncovered that a lncRNA, named cardiac apoptosis-related lncRNA (CARL), had capability to suppress mitochondrial fission and apoptosis by targeting miR-539 and PHB2, which participated in post myocardial infarction remodeling (5). Moreover, lncRNA-ROR was confirmed modulating expression of ANP and BNP in cardiomyocytes by means of sequestering miR-133 (6). Although several detailed ncRNAs have been identified associated with cardiovascular biology, more regulatory paths and promising targets are still needed for further clinical validation, and studying from a regulatory network perspective is a novel and integrated approach.

In the present study, we used TAC operated mice, which have undergone cardiac hypertrophic and ventricular remodeling processes, to imitate pressure overload induced HF. We investigated comprehensive expression profiles of lncRNAs, miRNAs and mRNAs based on microarray data between HF and control group. In addition, we performed integrated analysis of differentially expressed lncRNAs, miRNAs and mRNAs to identify gene functions and pathways involved in HF. Finally, we constructed a lncRNAmiRNA-mRNA competing regulatory network based on predicted RNA interactions and their expression patterns. Our results suggested that lncRNAs, miRNAs and mRNAs express distinctly in HF and are potential biomarkers and regulatory targets, which blazed new trails to early diagnosis and novel therapy for HF.

We present the following article in accordance with the ARRIVE reporting checklist (available at http://dx.doi. org/10.21037/cdt-20-465).

\section{Methods}

\section{Establishment of HF mouse model and sample acquisition}

Male C57/BL6 mice of SPF level rendered by Experimental Animal Center of Guangzhou University of Chinese Medicine (No. 44005800003866), were randomly divided into two groups: the HF group $(n=3)$ and the Sham group $(n=3)$, which were matched by age, weight and health state. The HF group underwent TAC operation which was a modification as previously described $(7,8)$, while the Sham group underwent a similar surgery but without aortic arch constriction. Experiments were performed under a project license (No. 2013081A) granted by ethics board of Guangdong Provincial People's Hospital, in compliance with the national and institutional guidelines for the care and use of animals. Four weeks after operation, with transthoracic echocardiography having confirmed the occurrence of HF in HF group, all mice were sacrificed and hearts were removed. Body weight and heart weight were measured and noted down. Gross views of hearts were photographed. Parts of heart tissue were performed HE and Masson staining and observed under microscope. The rest of heart tissues, which were used for microarray detection, were immediately soaked in RNA later stabilization resolution and stored in liquid nitrogen.

\section{Acquisition of microarray data}

Detection services of Arraystar Mouse LncRNA Microarray V3.0 and Exiqon microRNA Microarray 19.0 were performed by Kangchen Biotechnology Co., Ltd (Shanghai, China). Total RNA was extracted from each sample using TRIzol reagent (Invitrogen) following the product instructions. RNA quantity and quality were measured by NanoDrop ND-1000, and RNA integrity was assessed by standard denaturing agarose gel electrophoresis. Sample 
Table 1 Echocardiography data in each group $\bar{x} \pm S D$

\begin{tabular}{lcc}
\hline Parameter & Sham group $(n=3)$ & HF group $(n=3)$ \\
\hline LVEDD $(\mathrm{mm})$ & $3.36 \pm 0.17$ & $4.29 \pm 0.16^{*}$ \\
LVESD $(\mathrm{mm})$ & $2.33 \pm 0.27$ & $3.48 \pm 0.23^{*}$ \\
EF $(\%)$ & $59.08 \pm 6.92$ & $39.22 \pm 4.13^{*}$ \\
FS $(\%)$ & $30.60 \pm 4.66$ & $18.87 \pm 2.23^{*}$ \\
HR $(\mathrm{bpm})$ & $549.10 \pm 40.48$ & $585.00 \pm 60.53$ \\
\hline
\end{tabular}

*, $\mathrm{P}<0.05$ vs. Sham group. LVEDD, left ventricular end-diastolic diameter; LVESD, left ventricular end-systolic diameter; EF, ejection fraction; FS, fractional shortening; HR (bpm), heart rate (beats per minute); HF, heart failure.

preparation and microarray hybridization were performed according to the manufacturer's standard protocols with minor modifications. After array image collection, quantile normalization and subsequent data processing, lncRNA, miRNA and mRNA expression data were acquired for further analysis.

\section{qPCR verification}

In order to assess the reliability of microarray results, several differentially expressed lncRNAs, miRNAs and mRNAs were randomly selected for quantitative real-time PCR (qPCR). All reactions were performed independently in triplicate. U6, $\beta$-actin and GAPDH were used as internal references. Relative expression level was calculated using the $2^{-\Delta \Delta C \mathrm{t}}$ method. Primer sequences used for each test were listed in supplementary material (Table S1).

\section{Identification of DElncRNAs, DEmiRNAs and DEmRNAs}

Student's $t$-test was conducted when making comparisons between HF group and Sham group. DEmiRNAs and DEmRNAs were defined as miRNAs and mRNAs with "absolute fold change $\geq 2$ and $\mathrm{P}$ value $<0.05$ ". DElncRNAs were defined as $\operatorname{lncRNAs}$ with "absolute fold change $\geq 1.5$ and $\mathrm{P}$ value $<0.05$ " as previous studies $\operatorname{did}(9,10)$.

\section{GO enrichment analysis and patbway analysis of DEmRNAs}

GO is a major bioinformatics tool to annotate genes and analyze biological processes of genes (11). GO enrichment analysis were performed using an online tool DAVID (The Database for Annotation, Visualization and Integrated
Discovery, http://david.ncifcrf.gov) (version 6.8) $(12,13)$. Pathway enrichment analysis was performed using KEGG database (14) and Panther database (15).

\section{ceRNA regulatory network construction}

We constructed the competing endogenous RNA regulatory network based on interactions among lncRNAs, miRNAs and mRNAs, which can be predicted by databases. Interactions between lncRNA and miRNA were predicted by ENCORI $(16,17)$. And Interactions between miRNA and mRNA were retrieved from miRTarBase (http:// mirtarbase. mbc. nctu. edu. tw/php/index. php) which is an online database providing experimentally validated microRNA-target interactions (18). According to the competing regulation theory, the paired lncRNA and miRNA have opposite expressions, as well as the paired miRNA and mRNA. Thus, we took RNA expression patterns into consideration, together with their predicted interactions, to construct the ceRNA regulatory network.

\section{Statistical analysis}

Numerical data were presented as mean \pm SD. Student's $t$-test was performed for comparison between two groups. All statistics were processed using GraphPad Prism 8.0.2 and SPSS 22.0 software. $\mathrm{P}<0.05$ was considered statistically significant.

\section{Results}

\section{HF mouse model}

Four weeks after TAC operation, echocardiography showed that mice in HF group developed ventricular enlargement and HF compared with those in Sham group (Table 1).

From gross views of hearts, in contrast to Sham group, the sizes and outer diameters of hearts in HF group were obviously increased. Heart/body weight ratios were also increased in HF group (Table S2). HE staining showed a change of myocardial structural remodeling and a decrease of cardiomyocyte density in HF group. Masson staining suggested fibrotic changes in myocardial tissue of HF group (Figure 1), as collagen fibers were dyed blue.

Echocardiography, gross view of heart and histological alterations of heart tissue consistently confirmed the HF condition of mice in HF group. The pressure overload induced HF model was established successfully. 


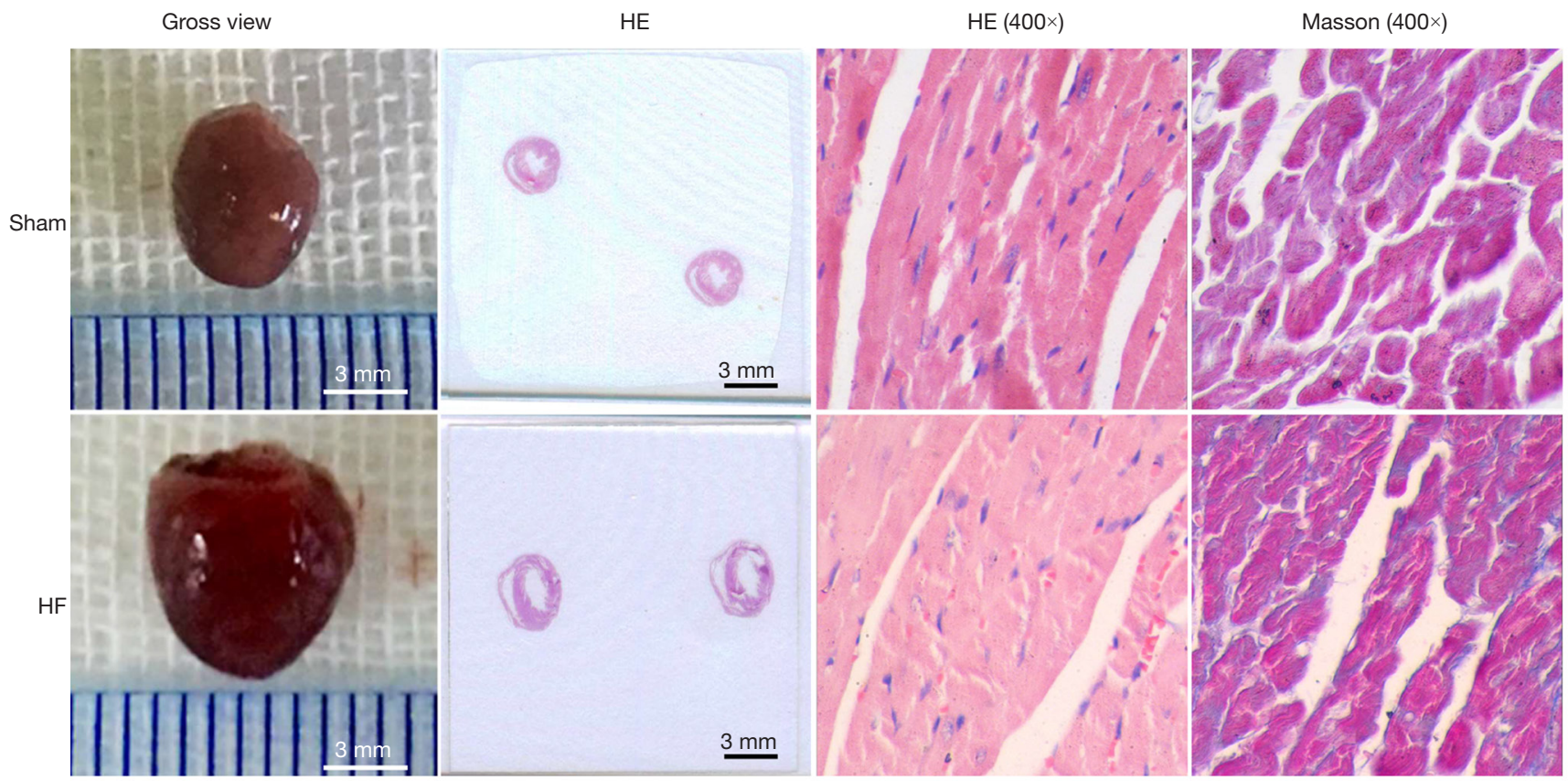

Figure 1 Gross view and histological observation of hearts. HF, heart failure; HE, hematoxylin-eosin staining.
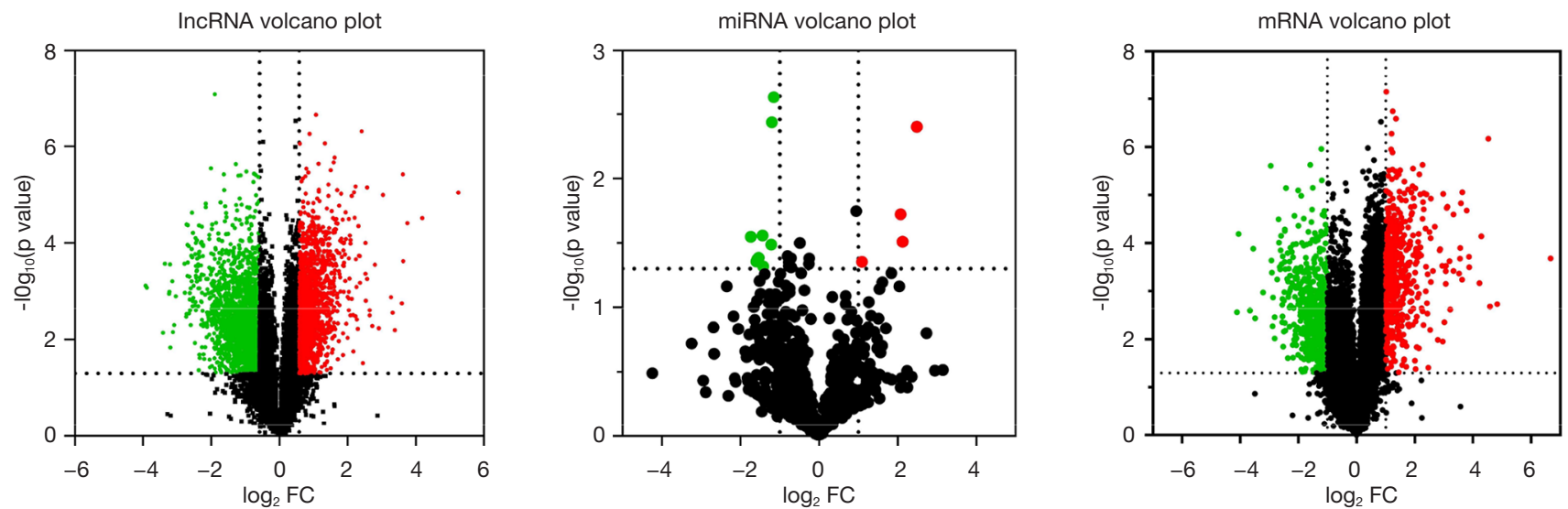

Figure 2 Volcano plots of lncRNA, miRNA and mRNA transcript expressions. Red color represents up regulation. Green color represents down-regulation.

\section{Identification of DElncRNAs, DEmiRNAs and DEmRNAs}

By means of volcano plotting with already set screening criteria, 3,830 DElncRNAs (including 1,707 up-regulated and 2,123 down-regulated DElncRNAs), 13 DEmiRNAs (including 4 up-regulated and 9 down-regulated DEmiRNAs) and 1,139 DEmRNAs (including 535 upregulated and 604 down-regulated DEmRNAs) were identified (Figure 2).

Hierarchical clustering of 1,139 DEmRNAs was conducted using $\mathrm{MeV}$ (Figure 3), which showed that DEmRNAs could basically differentiate HF group from Sham group.

\section{Verification of microarray results by $q P C R$}

Nine RNAs with three in DElncRNAs, DEmiRNAs and DEmRNAs respectively were randomly selected for qPCR detection, which aimed to test the reliability of microarray 


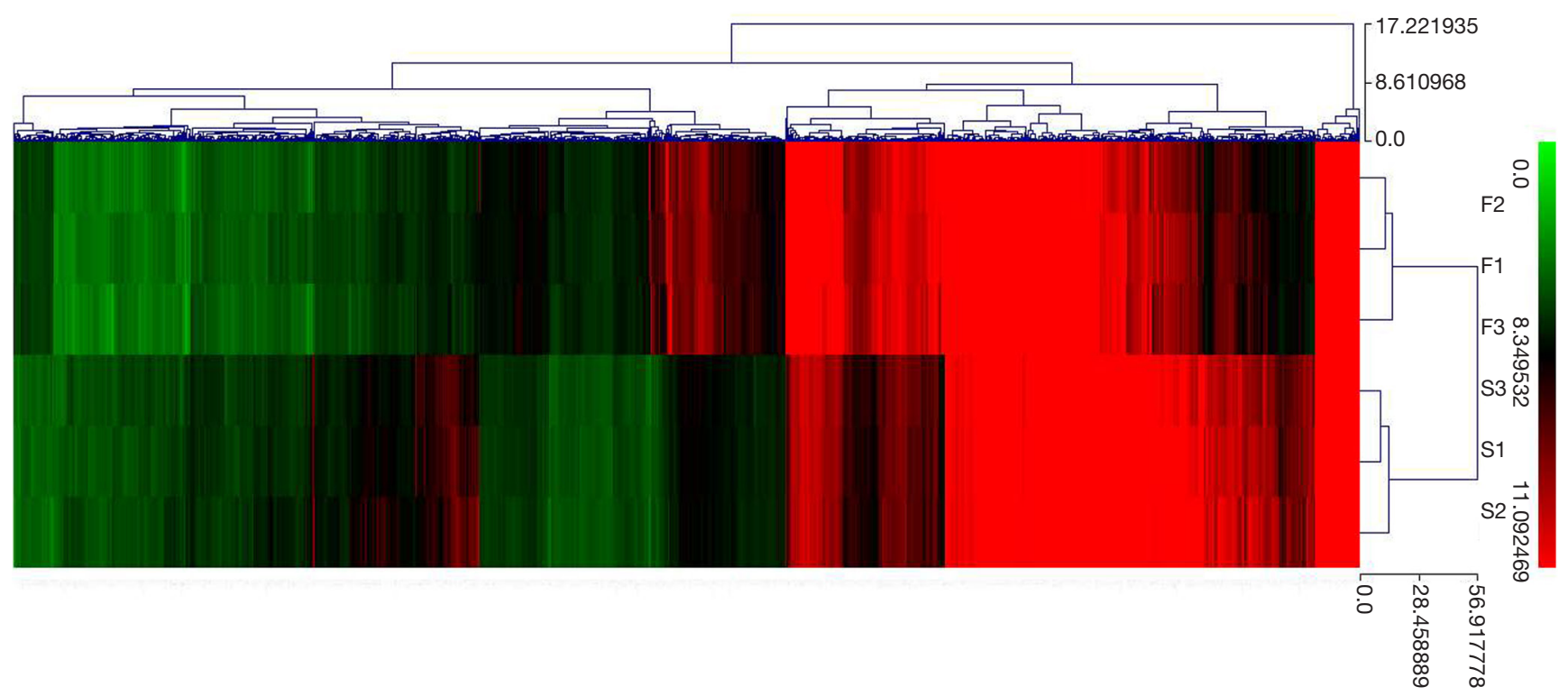

Figure 3 Hierarchical clustering of 1,139 differentially expressed mRNAs. F1, F2 and F3 represent different samples in HF group; S1, S2 and $\mathrm{S} 3$ represent different samples in Sham group.

data. qPCR results showed considerable consistency to microarray data, as 8 of 9 detected RNAs displayed similar significant differential level to their microarray results, except for mRNA Wnt10b which was nearly 5 folds down regulated in HF compared to Sham group but showed no statistical significance with $\mathrm{P}=0.071$ slightly higher than 0.05 (Figure 4). Basically, microarray data was confirmed reliable by qPCR, which could be used for further analysis.

\section{GO and pathway enrichment analysis of DEmRNAs}

The top ten most enriched GO terms for each category were shown in a chart (Figure 5). The most enriched GO terms for MF, CC and BP category respectively were extracellular matrix structural constituent, proteinaceous extracellular matrix and positive regulation of apoptotic process.

Pathway enrichment analysis was performed simultaneously by both KEGG database and Panther database (Figure 6). Among KEGG pathways, proteoglycans in cancer was the most significantly enriched pathway. For Panther pathway analysis, integrin signaling pathway was the only one significantly enriched pathway.

\section{ce $R N A$ regulatory network construction}

According to miRNA-lncRNA interactions predicted by
ENCORI, of the 13 DEmiRNAs and 3,830 DElncRNAs, 22 DElncRNAs could probably interact with 5 DEmiRNAs (Table 2). In the light of miRTarBase prediction, among the total 13 DEmiRNAs and 1,139 DEmRNAs, 3 DEmiRNAs could probably target 25 DEmRNAs (Table 3). Based on predicted interactions and expression patterns that two mutually targeted RNAs are negatively regulated, the competing endogenous regulatory network of RNA was constructed. Only DElncRNAs, DEmiRNAs and DEmRNAs that formed complete lncRNA-miRNA-mRNA competing regulatory axis were retained in the network. At last, 5 DElncRNAs, 3 DEmiRNAs and 16 DEmRNAs built up a ceRNA regulatory network (Figure 7).

\section{Discussion}

$\mathrm{HF}$ is a merging result of various heart diseases, which presents poor prognosis. Researches on its mechanism and therapy are under tense demand. A growing number of ncRNAs have been found associated with cardiovascular diseases (19-22), the regulatory mode of these ncRNAs is yet poorly understood. Cardiac hypertrophy is widely accepted as one of the crucial pathophysiological response to weaken cardiac contraction in a long term and eventually promote HF, which is characterized by enlargement of cardiomyocytes and accumulation of extracellular matrix. 


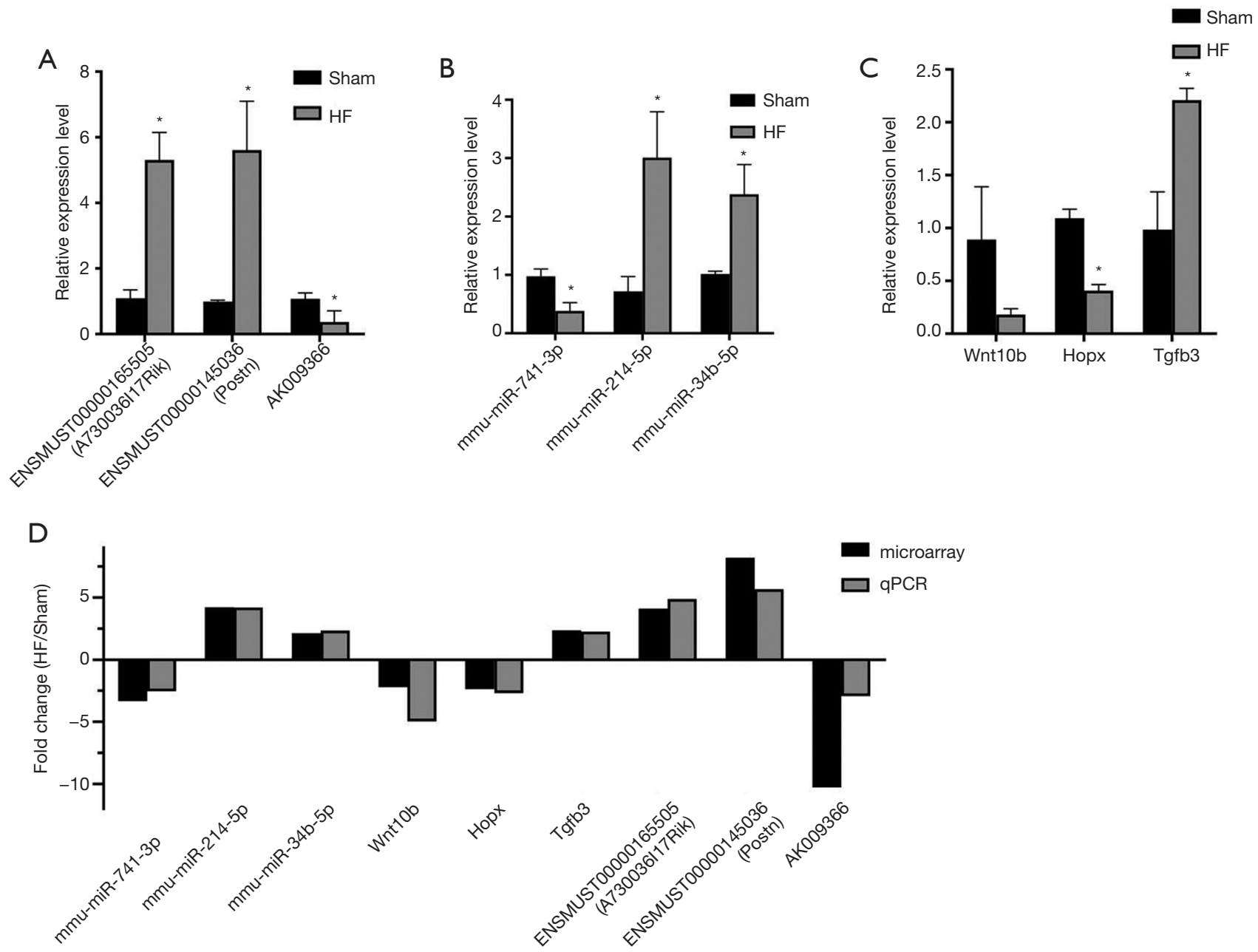

Figure 4 qPCR verification result. (A,B,C) qPCR results of selected miRNAs, mRNAs and lncRNAs; (D) comparison of RNA expression fold change between qPCR and microarray method; * $\mathrm{P}<0.05$ compared with Sham group; qPCR, quantitative polymerase chain reaction; $\mathrm{HF}$, heart failure.

miR-208 was the first reported pro-hypertrophic miRNA, whose regulatory role on cardiac function have been confirmed in transgenic mouse model (3). Besides, in Wang's work (4), lncRNA CHRF (cardiac hypertrophyrelated factor) was discovered capable of inducing hypertrophy through both in vivo and in vitro experiments. Therefore, ncRNAs are hopeful candidate targets for cardiac therapy. However, promising candidate targets are still few, and a small number of studies simultaneously investigated detailed lncRNA, miRNA and mRNA regulations in pressure overload induced HF. Therefore, our study is to examine comprehensive expression profiles of both lncRNA, miRNA and mRNAs in HF and their competing regulatory network.

In the present study, we successfully established $\mathrm{HF}$ mouse model using TAC method. Echocardiography showed that mice in HF group developed HF as they had enlarged ventricles and lower systolic function. Histological observation saw an occurrence of fibrosis and loss of cardiomyocytes in heart tissues of HF mice. lncRNA, miRNA and mRNA expression profiles between HF group and Sham group were obtained. Totally, 3,830 lncRNAs, 13 miRNAs and 1,139 mRNAs had significantly differential expressions between two groups, which together with heatmap of DEmRNAs suggested their potential as biomarkers to distinguish HF and provided clues for us 


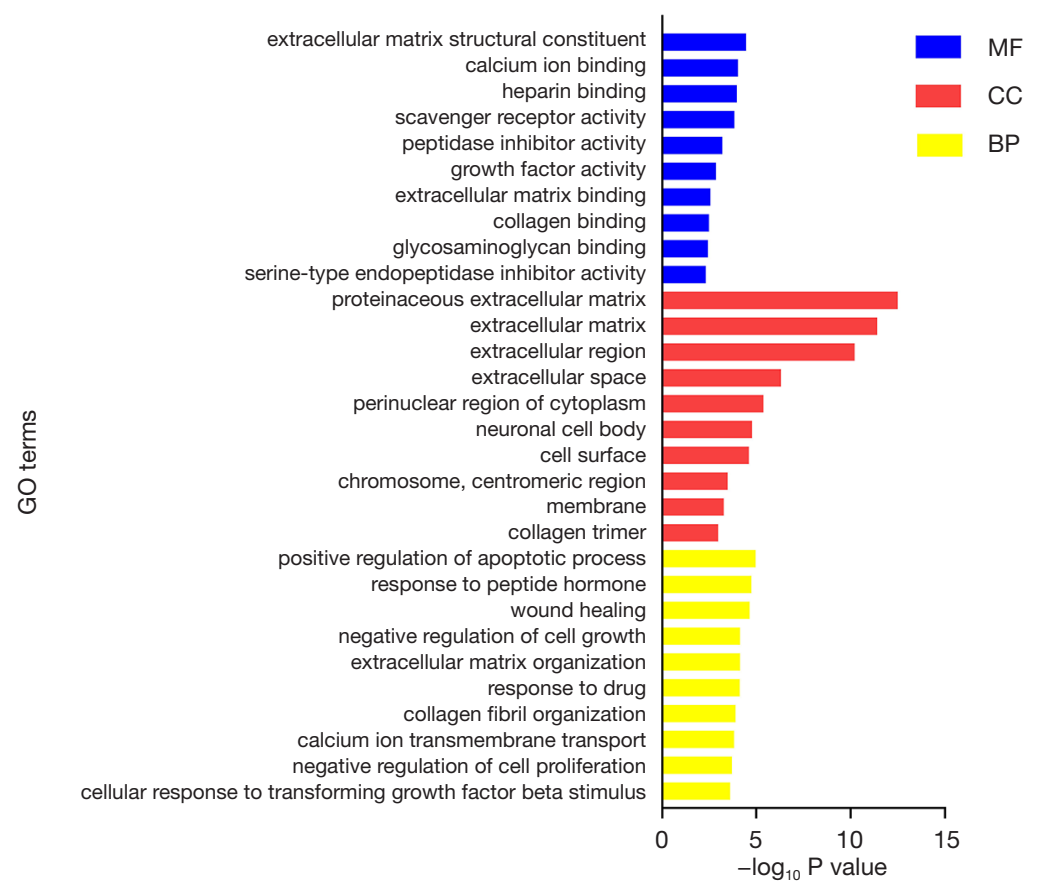

Figure 5 Top 10 most enriched terms of MF, CC and BP category in Gene Ontology enrichment analysis. MF, molecular function; CC, cellular component; BP, biological process.

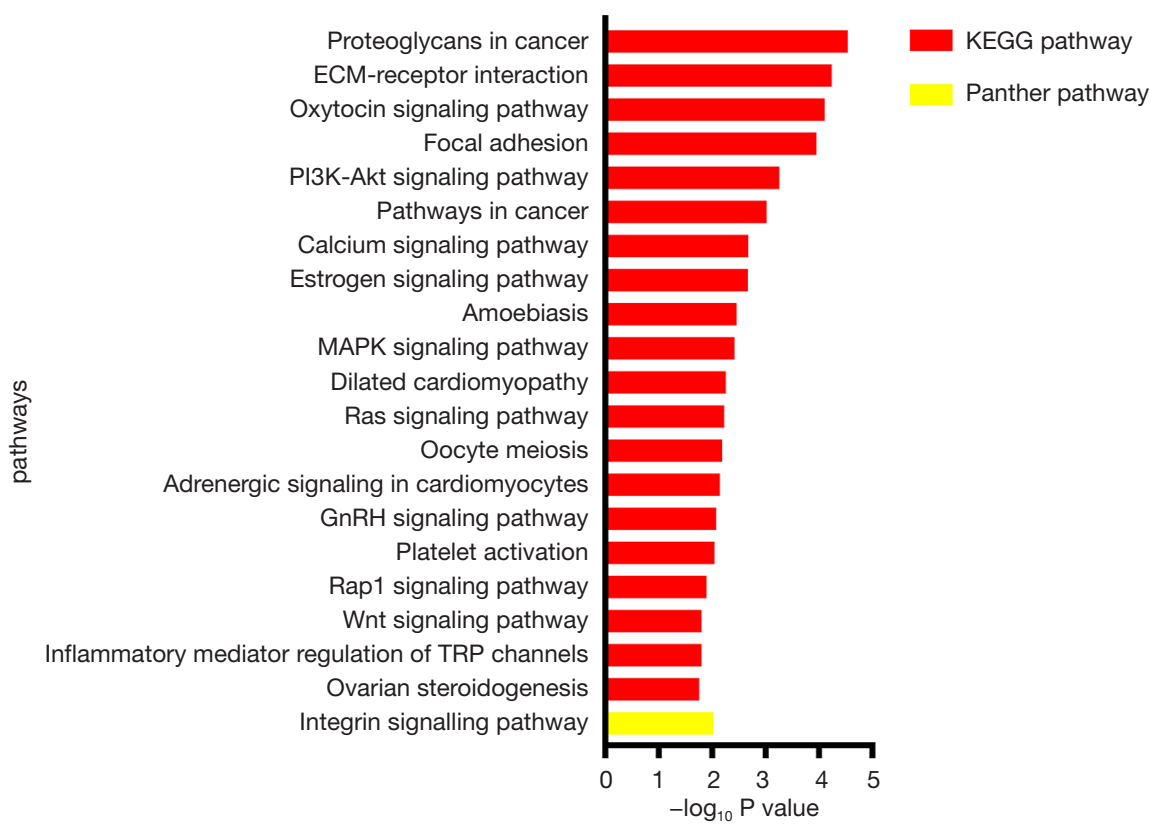

Figure 6 Top 20 significantly enriched KEGG pathways and the only one significantly enriched Panther pathway in pathway enrichment analysis of 1,139 differentially expressed mRNAs. KEGG, Kyoto Encyclopedia of Genes and Genomes database. 
Table 2 Putative DElncRNAs that may interact with DEmiRNAs

\begin{tabular}{ll}
\hline DEmiRNAs & DEIncRNAs (host gene) \\
\hline mmu-miR-34b-5p & ENSMUST00000124912 (Gm11280), ENSMUST00000168978 (Gm20427), ENSMUST00000113671 (Gm21986) \\
mmu-miR-214-5p & ENSMUST00000168978 (Gm20427) \\
mmu-miR-344b-5p & ENSMUST00000180984 (Gm3764), ENSMUST00000181396 (Gm3764), ENSMUST00000136359 (H19), \\
& ENSMUST00000239369 (Snhg5), ENSMUST00000128458 (Meg3), ENSMUST00000124106 (Meg3) \\
mmu-miR-741-3p & ENSMUST00000159890 (Gas5), ENSMUST00000160152 (Gas5), ENSMUST00000161380 (Gas5), \\
& ENSMUST00000162558 (Gas5), ENSMUST00000161461 (Gas5), ENSMUST00000174039 (1110038B12Rik), \\
& ENSMUST00000173811 (1110038B12Rik), ENSMUST00000172501 (1110038B12Rik), ENSMUST00000173070 \\
& (1110038B12Rik), ENSMUST00000127409 (5530601H04Rik), ENSMUST00000154334 (5530601H04Rik), \\
& ENSMUST00000156974 (5530601H04Rik), ENSMUST00000138460 (5530601H04Rik) \\
& ENSMUST00000159890 (Gas5), ENSMUST00000160152 (Gas5), ENSMUST00000161380 (Gas5), \\
& ENSMUST00000162558 (Gas5), ENSMUST00000161461 (Gas5) \\
\hline
\end{tabular}

DEIncRNAs, differentially expressed long noncoding RNA; DEmiRNAs, differentially expressed microRNAs.

Table 3 Putative interactions between DEmRNAs and DEmiRNAs

\begin{tabular}{ll}
\hline DEmiRNAs & DEmRNAs \\
\hline mmu-miR-34b-5p & Met, Cnnm3, Med22, Kcnq2, Kcna1, Insr, Dbn1, Pak1, Sfmbt1, Gdap1, Sox4, Edem3, Dclk1, Rab15, Nf1, Dtl, \\
& Mif4gd, Mical3, Gprin3 \\
mmu-miR-741-3p & Fam161b, St8sia3, Vav1, Bloc1s6 \\
mmu-miR-1298-5p & Loxl2, Hopx
\end{tabular}

DEmRNAs, differentially expressed messenger RNAs; DEmiRNAs, differentially expressed microRNAs.

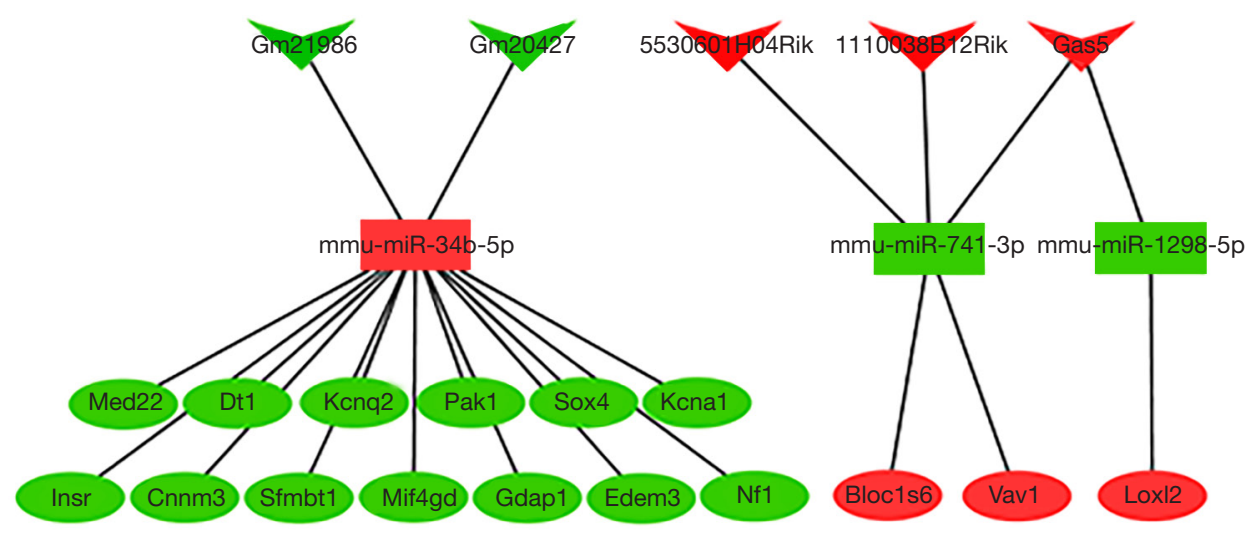

Figure 7 competing endogenous RNA regulatory network of DElncRNAs, DEmiRNAs and DEmRNAs. The V-shaped, rectangle and elliptical nodes represent DElncRNAs, DEmiRNAs and DEmRNAs respectively. Red and green color represent up regulation and down regulation respectively.

to further explore the complex regulatory mechanisms underlying $\mathrm{HF}$.

To have a general understanding of functions of differentially expressed genes in $\mathrm{HF}$, we perform GO and
KEGG pathway analysis on 1,139 DEmRNAs, as well as Panther pathway analysis. GO analysis showed that genes were significantly enriched in positive regulation of apoptotic process, response to peptide hormone, wound 
healing, proteinaceous extracellular matrix, extracellular matrix, extracellular region, extracellular matrix structural constituent, calcium ion binding, heparin binding, etc. Pathway analysis had similar results. Proteoglycans in cancer, ECM-receptor interaction and Oxytocin signaling pathway were the top three most enriched KEGG pathways, while integrin signaling pathway was the only one significant enriched Panther pathway. The enriched GO terms of BP, CC and MF category referred to several crucial pathophysiological processes in HF, such as extracellular matrix accumulation, apoptosis or cell cycle of cardiomyocyte and calcium ion transportation, which are widely accepted biological alterations in HF (23). Pathway analysis also echoed the GO analysis results as ECMreceptor interaction, apoptosis related PI3K-Akt signaling pathway and adrenergic signaling in cardiomyocytes (a contraction related pathway) were among enriched KEGG pathways and the fibrosis related integrin signaling pathway was the single enriched Panther pathway.

This study was intended to explore modulatory mechanisms in HF from a ceRNA perspective, in an attempt to establish a ceRNA regulatory network. In total, 5 DElncRNAs, 3 DEmiRNAs and 16 DEmRNAs were used to construct the ceRNAs regulatory network which contained 33 lncRNA-miRNA-mRNA regulatory axis. Protein coding genes are executioners of biological processes, therefore functions of mRNAs basically decide the role of this regulatory network. Among the 16 mRNAs in the network, most of them had been reported participating in processes of cardiovascular diseases. Hopx, homeodomain-only protein, is highly expressed and indispensable in the developing heart, and exerts cardiogenesis regulation through interacting with serum response factor (SRF) and modulating SRF-dependent cardiac-specific gene expression (24). Hopx was significantly down-regulated in our study, which may to some extent disturbed cardiac-specific gene expressions and promote impaired cardiac function. NF1, neurofibromatosis-related protein NF-1, acts as a component of RAS pathway, which is within signaling networks that modulate proliferation, differentiation, cell survival and molecular control of energy homeostasis. Inactivation of neurofibromin 1, coding protein of NF-1, can cause type 1 neurofibromatosis (NF1), which presents cardiac malformations (25). In our study, NF1 had differentially lower expression in HF, which may lead to the cardiac abnormality in pressure overload condition. KCNA1, potassium voltage-gated channel subfamily A member 1, was a differentially low-expressed gene in this study. Glasscock found that KCNA1 deficiency leads to impaired neural control of cardiac rhythmicity, which can explain human sudden death in epilepsy (26). Thus, low expression of KCNA1 in HF partially accounted for arrhythmia in end-stage HF. Whereas there are still other genes in our ceRNA network which have not been reported in heart diseases, such as Fam161b, Vav1, Bloc1s6, etc. The function of these genes in cardiovascular system is still yet to discover and needs further effort of exploration. Depending on functions of mRNAs, the ceRNA regulatory network hopefully undertakes a crucial part in HF.

lncRNAs, miRNAs and mRNAs were linked in the network according to ceRNA regulation theory. During previous exploration of CHRF/miR-489/Myd88 axi (4), lncRNA CHRF and miR-489 were negatively associated in hypertrophic cardiomyocyte, as well as miR-489 and My88, which provided a clue for further investigation. Through luciferase report experiments confirming their mutual interactions and further validation tests, it was finally assured that CHRF regulates hypertrophy through sponging miR-489 which targets pro-hypertrophic gene Myd88. In the ceRNA network of our study, 5 lncRNAs (Gm20427, Gm21986, 1110038B12Rik, 5530601H04Rik and Gas5) and 3 miRNAs (mmu-miR-34b-5p, mmu-miR-741-3p and mmu-miR-1298-5p) were putative upstream molecules of mRNAs. Among them, lncRNA Gas5 has been widely noted to participate in myocardial ischemia/reperfusion injury and atherosclerosis progression, down regulation of which can improve cardiac function in ill condition $(27,28)$. In our study lncRNA Gas5 was up-regulated in HF, which conformed to the previous report. What's more, miR$34 b-5 p$, a significantly up-regulated miRNA, has been reported associated with various processes, such as agerelated vascular dysfunction, pulmonary fibrosis, skeletal muscle formation and tumorigenesis (29-32). Whether lncRNA Gas5 and miR-34b-5p execute the aforementioned functions as ceRNAs in a competing regulatory way is a research direction that our study provided. Generally, the network in our study provided preliminary regulation paths and molecular connectivity to guide further research, functional experiments are in demand to clarify.

To date, RNA therapy is seeing a bright future. There are a considerable number of drugs targeting RNAs to treat specific diseases, which are based on their regulatory effect. For instance, Mipomersen, brand-named Kynamro, was approved to use in familial hypercholesterolemia, which decreases cholesterol level by targeting ApoB mRNA and inducing its degradation by RNase H (33). In addition, 
there are others under clinical trial, such as Miravirsen targeting miR-122 to treat Hepatitis C and MRG-106 targeting miR-155 used in lymphomas (34). Similarly, RNA diagnosis is evolving as a growing number of RNAs are showing high diagnostic efficiency $(35,36)$. Our study filtrating hopeful targets and RNA biomarkers of HF were expected to add more to current researches.

The expression profiles of RNAs and their interactions shown in our study were only a screening process based on experimental data using bioinformatics analyses. The TAC model of HF based on pressure overload, cannot cover all subtypes of HF, such as volume overload and tachycardia induced HF, etc., which is a limitation of this study. Thus, to explore more comprehensive and deeper insights, different HF models and more HF samples are required. Validation experiments are also needed to perform both in vivo and in vitro to verify our findings.

\section{Conclusions}

To conclude, the present study identified a total of 3,830 DElncRNAs, 13 DEmiRNAs and 1,139 DEmRNAs, which were hopeful diagnostic biomarkers and might be involved in the pathogenesis or progression of HF. A ceRNA regulatory network containing 5 DElncRNAs, 3 DEmiRNAs and 16 DEmRNAs was established, which provided candidate target paths for HF therapy. Further studies are needed to testify our findings.

\section{Acknowledgments}

Funding: This study was supported by National Natural Science Foundation of China (Project Number: 81470571) and National Science Foundation for Young Scientists of China (Project Number: 81300230).

\section{Footnote}

Reporting Checklist: The authors present the study in accordance with the ARRIVE reporting checklist. Available at http://dx.doi.org/10.21037/cdt-20-465

Data Sharing Statement: Available at http://dx.doi. org/10.21037/cdt-20-465

Peer Review File: Available at http://dx.doi.org/10.21037/ cdt-20-465
Conflicts of Interest: All authors have completed the ICMJE uniform disclosure form (available at http://dx.doi. org/10.21037/cdt-20-465). The authors have no conflicts of interest to declare.

Ethical Statement: The authors are accountable for all aspects of the work in ensuring that questions related to the accuracy or integrity of any part of the work are appropriately investigated and resolved. Experiments were performed under a project license (No. 2013081A) granted by ethics board of Guangdong Provincial People's Hospital, in compliance with the national and institutional guidelines for the care and use of animals.

Open Access Statement: This is an Open Access article distributed in accordance with the Creative Commons Attribution-NonCommercial-NoDerivs 4.0 International License (CC BY-NC-ND 4.0), which permits the noncommercial replication and distribution of the article with the strict proviso that no changes or edits are made and the original work is properly cited (including links to both the formal publication through the relevant DOI and the license). See: https://creativecommons.org/licenses/by-nc-nd/4.0/.

\section{References}

1. Shafazand M, Schaufelberger M, Lappas G, et al. Survival trends in men and women with heart failure of ischaemic and non-ischaemic origin: data for the period 1987-2003 from the Swedish Hospital Discharge Registry. Eur Heart J 2009;30:671-8.

2. Thomson DW, Dinger ME. Endogenous microRNA sponges: evidence and controversy. Nat Rev Genet 2016;17:272-83.

3. Dangwal S, Schimmel K, Foinquinos A, et al. Noncoding RNAs in Heart Failure. Handb Exp Pharmacol 2017;243:423-45.

4. Wang K, Liu F, Zhou L, et al. The Long Noncoding RNA CHRF Regulates Cardiac Hypertrophy by Targeting miR489. Circ Res 2014;114:1377-88.

5. Wang K, Long B, Zhou L, et al. CARL lncRNA inhibits anoxia-induced mitochondrial fission and apoptosis in cardiomyocytes by impairing miR-539-dependent PHB2 downregulation. Nat Commun 2014;5:3596.

6. Jiang F, Zhou X, Huang J. Long Non-Coding RNA-ROR Mediates the Reprogramming in Cardiac Hypertrophy. PLoS One 2016;11:e0152767.

7. Xiao CY, Chen M, Zsengellér Z, et al. Poly(ADP-Ribose) 
Polymerase Promotes Cardiac Remodeling, Contractile Failure, and Translocation of Apoptosis-Inducing Factor in a Murine Experimental Model of Aortic Banding and Heart Failure. J Pharmacol Exp Ther 2005;312:891-8.

8. Arany Z, Novikov M, Chin S, et al. Transverse aortic constriction leads to accelerated heart failure in mice lacking PPAR-coactivator 1. Proc Natl Acad Sci U S A 2006;103:10086-091.

9. Cao Y, Yang Y, Wang L, et al. Analyses of long non-coding RNA and mRNA profiles in right ventricle myocardium of acute right heart failure in pulmonary arterial hypertension rats. Biomed Pharmacother 2018;106:1108-15.

10. Zhang J, Fan D, Jian Z, et al. Cancer Specific Long Noncoding RNAs Show Differential Expression Patterns and Competing Endogenous RNA Potential in Hepatocellular Carcinoma. PLoS One 2015;10:e0141042.

11. Ashburner M, Ball CA, Blake JA, et al. Gene Ontology: tool for the unification of biology. Nat Genet 2000;25:25-9.

12. Huang da W, Sherman BT, Lempicki RA. Systematic and integrative analysis of large gene lists using DAVID bioinformatics resources. Nat Protoc 2009;4:44-57.

13. Huang da $W$, Sherman BT, Lempicki RA. Bioinformatics enrichment tools: paths toward the comprehensive functional analysis of large gene lists. Nucleic Acids Res 2009;37:1-13.

14. Kanehisa M. The KEGG database. Novartis Found Symp 2002;247:91-101; discussion 101-3, 119-28, 244-52.

15. Mi H, Muruganujan A, Ebert D, et al. PANTHER version 14: more genomes, a new PANTHER GO-slim and improvements in enrichment analysis tools. Nucleic Acids Res 2019;47:D419-D426.

16. Li JH, Liu S, Zhou H, et al. starBase v2.0: decoding miRNA-ceRNA, miRNA-ncRNA and protein-RNA interaction networks from large-scale CLIP-Seq data. Nucleic Acids Res 2014;42:D92-D97.

17. Fan K, Zebisch A, Horny K, et al. Highly Expressed miR-375 is not an Intracellular Oncogene in Merkel Cell Polyomavirus-Associated Merkel Cell Carcinoma. Cancers 220;12:529.

18. Chou CH, Shrestha S, Yang C, et al. miRTarBase update 2018; a resource for experimentally validated microRNA-target interactions. Nucleic Acids Res 2018;46:D296-D302.

19. Zangrando J, Zhang L, Vausort M, et al. Identification of candidate long non-coding RNAs in response to myocardial infarction. BMC Genomics 2014;15:460.

20. Yang KC, Yamada KA, Patel AY, et al. Deep RNA
Sequencing Reveals Dynamic Regulation of Myocardial Noncoding RNAs in Failing Human Heart and Remodeling With Mechanical Circulatory Support. Circulation 2014;129:1009-21.

21. Saddic LA, Sigurdsson MI, Chang T, et al. The long noncoding RNA landscape of the ischemic human left ventricle. Circ Cardiovasc Genet. 2017;10:e001534.

22. Enes Coşkun M, Kervancıoğlu M, Öztuzcu S, et al. Plasma microRNA profiling of children with idiopathic dilated cardiomyopathy. Biomarkers 2016;21:56-61.

23. Liew CC, Dzau VJ. Molecular genetics and genomics of heart failure. Nat Rev Genet 2004;5:811-25.

24. Chen F, Kook H, Milewski R, et al. Hop is an unusual homeobox gene that modulates cardiac development. Cell 2002;110:713-23.

25. Dard L, Bellance N, Lacombe D, et al. RAS signaling in energy metabolism and rare human diseases. Biochim Biophys Acta Bioenerg 2018;1859:845-67.

26. Glasscock E, Yoo JW, Chen TT, et al. Kv1.1 Potassium Channel Deficiency Reveals Brain-Driven Cardiac Dysfunction as a Candidate Mechanism for Sudden Unexplained Death in Epilepsy. J Neurosci 2010;30:5167-75.

27. Meng XD, Yao HH, Wang LM, et al. Knockdown of GAS5 Inhibits Atherosclerosis Progression via Reducing EZH2-Mediated ABCA1 Transcription in ApoE-/- Mice. Mol Ther Nucleic Acids 2020;19:84-96.

28. Wu N, Zhang X, Bao Y, et al. Down-regulation of GAS5 ameliorates myocardial ischaemia/reperfusion injury via the miR-335/ROCK1/AKT/GSK-3 axis. J Cell Mol Med 2019;23:8420-31.

29. Hu RP, Lu YY, Zhang XJ. MiR-34b-5p knockdown attenuates bleomycin-induced pulmonary fibrosis by targeting tissue inhibitor of metalloproteinase 3 (TIMP3). Eur Rev Med Pharmacol Sci 2019;23:2273-9.

30. Li H, Wang X, Lu X, et al. Co-expression network analysis identified hub genes critical to triglyceride and free fatty acid metabolism as key regulators of age-related vascular dysfunction in mice. Aging 2019;11:7620-38.

31. Wang Z, Zhang X, Li Z, et al. MiR-34b-5p Mediates the Proliferation and Differentiation of Myoblasts by Targeting IGFBP2. Cells 2019;8:360.

32. Dong L, Chen F, Fan Y, et al. MiR-34b-5p inhibits cell proliferation, migration and invasion through targeting ARHGAP1 in breast cancer. Am J Transl Res 2020;12:269-80.

33. Kim YK. RNA Therapy: Current Status and Future Potential. Chonnam Med J 2020;56:87-93. 
34. Zhou LY, Qin Z, Zhu YH, et al. Current RNA-based Therapeutics in Clinical Trials. Curr Gene Ther 2019;19:172-96.

35. Deng J, Tang J, Wang G, et al. Long non-coding RNA as potential biomarker for prostate cancer: is it making a

Cite this article as: He Y, Feng Z, Lu J, Wang R, Huang C, Zhou Y. Exploring biomarkers and therapeutic targets for pressure overload induced heart failure based on microarray data. Cardiovasc Diagn Ther 2020;10(5):1226-1237. doi: 10.21037/ cdt-20-465 difference? Int J Environ Res Public Health 2017;14:270.

36. E S, Costa MC, Kurc S, et al. The circulating non-coding RNA landscape for biomarker research: lessons and prospects from cardiovascular diseases. Acta Pharmacol Sin 2018;39:1085-99. 


\section{Supplementary}

Table S1 Primer sequences of RNAs for qPCR

\begin{tabular}{|c|c|}
\hline RNA & Primer sequence \\
\hline \multirow[t]{2}{*}{ GAPDH (MOUSE) } & F: 5'-CACTGAGCAAGAGAGGCCCTAT-3' \\
\hline & R: 5'-GCAGCGAACTTTATTGATGGTATT-3' \\
\hline \multirow[t]{2}{*}{ U6 } & F: 5'-GCTTCGGCAGCACATATACTAAAAT-3' \\
\hline & R: 5'-CGCTTCACGAATTTGCGTGTCAT-3' \\
\hline \multirow[t]{2}{*}{$\beta$-actin } & F: 5'-GTACCACCATGTACCCAGGC-3' \\
\hline & R: 5'-AACGCAGCTCAGTAACAGTCC-3' \\
\hline \multirow[t]{2}{*}{ mmu-miR-741-3p } & GSP: 5'-GGGGTGAGAGATGCCATTCTA-3' \\
\hline & R: 5'-GTGCGTGTCGTGGAGTCG-3' \\
\hline \multirow[t]{2}{*}{ mmu-miR-214-5p } & GSP: 5'-GGGCTGCCTGTCTACACTTG-3' \\
\hline & R: 5'-GTGCGTGTCGTGGAGTCG-3' \\
\hline \multirow[t]{2}{*}{ mmu-miR-34b-5p } & GSP: 5'-GGGGAAGGCAGTGTAATTAGC-3' \\
\hline & R: 5'-GTGCGTGTCGTGGAGTCG-3' \\
\hline \multirow[t]{2}{*}{ Wnt10b } & F: 5'-GCACTGTACTGTTAGCGTCTTCT-3' \\
\hline & R: 5'-CACAATGCCTGCTATTATCCTT-3' \\
\hline \multirow[t]{2}{*}{ Hopx } & F: 5'-CACCACGCTGTGCCTCAT-3' \\
\hline & R: 5'-GCGCTGCTTAAACCATTTCT-3' \\
\hline \multirow[t]{2}{*}{ Tgfb3 } & F: 5'-TTACTATGCCAACTTCTGCT-3' \\
\hline & R: 5'-CTGGGTTCAGGGTGTTGT-3' \\
\hline \multirow[t]{2}{*}{ ENSMUST00000165505 (A730036l17Rik) } & F: 5'-TCAACCTCACCAGCATTT-3' \\
\hline & R :5'-CTGCAGTGCCTTAATCTTC-3' \\
\hline \multirow[t]{2}{*}{ ENSMUST00000145036 (Postn) } & F: 5'-CTTGAGCGTCTCCAATCTGTA-3' \\
\hline & R: 5'-ACACCGTCATCCCTCGTTA-3' \\
\hline \multirow[t]{2}{*}{ AK009366 } & F: 5'-CACCTCTTCTGGCGTGTCTA3' \\
\hline & R: 5'-CTTGCTССTCСАСТССТTTा-3' \\
\hline
\end{tabular}

F, forward primer; R, reverse primer; GSP, gene specific primer.

Table S2 Ratio of heart weight/body weight in different groups $(\bar{x} \pm S D)$

\begin{tabular}{lcc}
\hline Parameter & Sham group $(\mathrm{n}=3)$ & HF group $(\mathrm{n}=3)$ \\
\hline BW $(\mathrm{g})$ & $24.73 \pm 1.96$ & $24.03 \pm 1.63$ \\
HW $(\mathrm{mg})$ & $105.33 \pm 13.99$ & $203.07 \pm 10.21^{\star}$ \\
HW/BW $(\mathrm{mg} / \mathrm{g})$ & $4.25 \pm 0.23$ & $8.5 \pm 1.03^{*}$ \\
\hline
\end{tabular}

*, $\mathrm{P}<0.05$ vs. Sham group. BW, body weight; HW, heart weight; HW/BW, heart weight/ body weight ratio; HF, heart failure. 